\title{
Dynamic Structure Factor of Ultracold Bosons in Optical Lattice
}

\author{
T.A. Zaleski* And T.K. KopeĆ \\ Institute of Low Temperature and Structure Research, Polish Academy of Sciences, \\ Okólna 2, 50-422 Wrocław, Poland
}

\begin{abstract}
Ultracold atoms in optical lattices have been intensively investigated in recent years as they provide a very well controllable environment for observation of many-body quantum phenomena, closely mimicking physics of strongly interacting electronic systems. Here, we use the quantum rotor approach supplemented by the Bogolyubov method to investigate one- and two-particle excitations, which are a measure of inter-particle correlations. We calculate one-particle spectral function and dynamic structure factor, which can be observed using spectroscopy of cold atomic systems. Our calculations require a significant numerical effort to determine multidimensional convolutions of momentum and frequency dependent constituents functions, which we achieve using parallelised fast Fourier transform. We observe the appearance of sharp coherence peaks in the superfluid phase of the cold bosons, which closely resembles the formation of sharply defined quasiparticle excitations below $T_{c}$ in cuprates or smeared excitation spectra characteristic for strongly interacting systems.
\end{abstract}

DOI: 10.12693 /APhysPolA.130.564

PACS/topics: 03.75.Hh, 05.30.Jp, 67.85.Hj

\section{Introduction}

The Bose-Einstein condensation (BEC) is a nontypical phase transition, which occurs in completely noninteracting system. It is governed purely by the quantum statistics of particles and as a result is a hallmark of quantum nature of matter. Urge to realize the BEC in a real system lead to development of cooling and trapping techniques of dilute atomic gases. This way proved to be successful and in nanokelvin temperature range the BEC was achieved, signalled by macroscopic occupation of the lowest, zero-momentum energy state. However, it was quickly realized that more precise trapping can be implemented and the ultracold atomic systems can be driven to a regime, in which they can simulate behaviour of strongly interacting lattice particles in very clean and controllable environment [1]. It can be accomplished using counter-propagating laser beams that localise atoms at maxima of the electromagnetic wave via AC Stark shift. Optical lattices allow for trapping bosons, fermions, mixtures, multicomponent particles, etc. Changing of parameters of trapping fields leads to very wide modification of atomic behaviour: a key phenomenon in these systems being a quantum phase transition between phase coherent superfluid state (SF) and strongly localized Mott insulator (MI), which is tuned by strength of interactions between atoms [2]. Geometry of the laser beams defines shape the lattice (dimensionality, structure), while their intensity controls ratio between hopping and on-site repulsion of particles. Although atoms in optical lattices are electrically neutral, they can still simulate behaviour of charged particles in

\footnotetext{
*corresponding author; e-mail: t.zaleski@int.pan.wroc.pl
}

magnetic field. This can be realized by direct imprinting of the geometric phase resulting from the presence of external synthetic magnetic field vector potential using rotation [1], photon-assisted processes [3] or lattice shaking via the Floquet schemes [4]. On the other hand, interactions between atoms can be modified (strength and type: attraction or repulsion) using the Feshbach resonance or simply by changing depth of the lattice potential. Finally, systems of arbitrary shaped can be engineered, in which atoms are allowed to occupy only selected locations e.g. implementing quantum contacts or quantum wires [5].

Properties of ultracold atoms can be investigated using number of probes. The most direct method allowing form determination of presence of the BEC is based on time-of-flight imaging. In these experiments, all trapping potentials are suddenly switched off, which temporarily freezes particle momenta. As a result, observation of the distribution of the atoms after arbitrary time allows for easy recovery of particle density as a function of momentum. As the BEC is connected with macroscopic occupation of the lowest energy state, it manifests itself with a sharp peak around zero-momentum in the time-of-flight experiments [2]. Furthermore, behaviour of atoms in optical lattices can be observed directly. Since, the lattice constant is of the order of half of wavelength of visible light, excited trapped atoms can be visible under optical microscope [6,7]. This allows for site-resolved analysis of atomic behaviour: site occupation statistics can determine the local ground state. In the opposite process, the microscope can be used to selectively shine light on selected lattice site, which is a way of controlling of local occupation, e.g. for building an initial state from which system evolution will be investigated [7]. On the other hand, the excitation spectrum 
of the atomic gases confined to optical lattices can be measured using methods based on response to scattering photons from the correlated atomic state. These include radio-frequency spectroscopy [8, 9], the Raman spectroscopy [10] and the Bragg spectroscopy [11-14]. These experiments, still not as mature as they solid-state equivalents (e.g. angle-resolved photoemission spectroscopy), can reveal the band structure of these systems.

Theoretical description of strongly interacting system is challenging due to a lack of a dominant energy scale. However, the quantum phase transition of the SF to the MI states, which is a central phenomenon in these systems, is governed by phase fluctuations as it occurs between phase coherent and strongly localised states. As a result, knowledge of the low-energy excited states is of highest importance: their simplest kinds involve one or two particles and are connected to the spectral function $A(\boldsymbol{k}, \omega)$ and dynamical structure factor $S(\boldsymbol{k}, \omega)$, respectively. Since these quantities are both momentum and frequency dependent, they require a theoretical description that properly includes spatial and thermal fluctuations. In the context of bosons, the dynamical structure factor was analysed mainly for low-dimensional systems, e.g. 1D [15-19]. Here, we use a combination of the quantum rotor approach and Bogolyubov theory to decouple problem of strongly correlated particles into formation of the superfluid amplitude and long-range phase coherence. This method allows us to calculate the dynamic structure factor of a two-dimensional bosonic lattice system in the regime of strong correlations. We implement the quantum rotor mapping of the Bose-Hubbard model [20] combined with the fast Fourier transform to track changes of $S(\boldsymbol{q}, \omega)$ in the momentum-energy space and identify signatures of the different quantum phases. The approach is supplemented with a significant numerical effort due to convolved nature of the problem. The remainder of the paper is as follows: in Sect. 2, we define the object of our studies. Furthermore, we provide a theoretical background and a brief presentation of our approach. In Sect. 4 we describe the numerical methods that we use and present obtained results. Finally, Sect. 5 is devoted to summary.

\section{Dynamic structure factor}

The dynamic structure factor [21] is defined as realtime density-density correlation function [22]:

$$
S(\boldsymbol{k}, \omega)=\sum_{\boldsymbol{r}} \mathrm{e}^{\mathrm{i} \boldsymbol{k} \boldsymbol{r}} \int \mathrm{d} t \mathrm{e}^{-\mathrm{i} \omega t}\left\langle\tilde{n}_{\boldsymbol{r}}(t) \tilde{n}(0)\right\rangle,
$$

where $\langle\ldots\rangle$ denotes the statistical averaging. The trivial contribution to $S(\boldsymbol{k}, \omega)$ at $\omega=0$ is excluded by considering $\tilde{n}_{\boldsymbol{r}}(t)=n_{\boldsymbol{r}}(t)-\left\langle n_{\boldsymbol{r}}(t)\right\rangle$ with $n_{\boldsymbol{r}}(t)$ being bosonic density. The dynamic structure factor can be measured by the Bragg spectroscopy [11-14]. The function $S(\boldsymbol{k}, \omega)$ contains information about correlations in the system obeying particle conservation and sum rules. The structure factor can be conveniently obtained using the Matsubara technique [23] from the "imaginary-time" $0<\tau<\beta\left(\beta=1 / k_{\mathrm{B}} T\right)$ density-density correlation function

$$
\chi(\boldsymbol{r}, \tau)=-\left\langle\tilde{n}_{\boldsymbol{r}}(\tau) \tilde{n}(0)\right\rangle .
$$

Continuation to real frequencies can be obtained employing the fluctuation-dissipation theorem

$$
S(\boldsymbol{k}, \omega)=\left.\frac{2 \operatorname{Im} \chi\left(\boldsymbol{k}, \omega_{m}\right)}{1-\mathrm{e}^{-\beta \omega}}\right|_{\mathrm{i} \omega_{m} \rightarrow \omega+\mathrm{i} 0^{+}},
$$

where $\omega_{m}=2 \pi m / \beta$ is the Matsubara frequency $(m=$ $0, \pm 1, \pm 2, \ldots)$. Finally, the imaginary part of the bosonic density-density correlation function (2) and its spectral density $A_{S}(\boldsymbol{k}, \omega)$ are related as follows:

$$
2 \operatorname{Im} \chi(\boldsymbol{k}, \omega)=A_{S}(\boldsymbol{k}, \omega) \text {. }
$$

Introducing this into Eq. (3) one arrives at

$$
S(\boldsymbol{k}, \omega)=\frac{A_{S}(\boldsymbol{k}, \omega)}{1-\mathrm{e}^{-\beta \omega}},
$$

which gives the momentum and energy dependent dynamic structure factor $S(\boldsymbol{k}, \omega)$.

\section{Model and method}

To analyse the behaviour of strongly correlated bosons in optical lattices usually we use the Bose-Hubbard model [24]:

$$
\begin{aligned}
\mathcal{H} & =-t \sum_{\left\langle\boldsymbol{r}>\boldsymbol{r}^{\prime}\right\rangle}\left(a_{\boldsymbol{r}}^{\dagger} a_{\boldsymbol{r}}^{\prime}+\text { H.c. }\right) \\
& +\frac{U}{2} \sum_{\boldsymbol{r}} n_{\boldsymbol{r}}\left(n_{\boldsymbol{r}}-1\right)-\mu \sum_{\boldsymbol{r}} n_{\boldsymbol{r}},
\end{aligned}
$$

where $a_{\boldsymbol{r}}^{\dagger}$ and $a_{\boldsymbol{r}}$ are the bosonic creation and destruction operators at a site $\boldsymbol{r}=1, \ldots, N$ of a two-dimensional square lattice, respectively. The ratio of $t$ being the hopping element and $U$ - the on-site interaction - drives the phase transition between MI and the superfluid state with long range phase coherence [25]. Finally, $n_{\boldsymbol{r}}$ is the particle number operator, while the chemical potential $\mu$ controls the total number of atoms in the lattice.

The main point of our method is a decomposition of the bosonic operators:

$$
a_{\boldsymbol{r}}(\tau)=\mathrm{e}^{\mathrm{i} \phi_{\boldsymbol{r}}(\tau)} b_{\boldsymbol{r}}(\tau),
$$

where $\phi_{\boldsymbol{r}}(\tau)$ is a fluctuating $\mathrm{U}(1)$ quantum rotor phase field, dual to the local bosonic density [26] and $b_{\boldsymbol{r}}(\tau)$ is a bosonic amplitude determining superfluid density. As a result, the on-site interaction in the Hamiltonian (6) is replaced in the Lagrangian of the model $L=$ $\int_{0}^{\beta} \mathrm{d} \tau\left(\mathcal{L}_{0}+\mathcal{L}^{\prime}\right)$ by a simple kinetic term $\mathcal{L}_{0}$ for the phase field,

$$
\mathcal{L}_{0}=\sum_{\boldsymbol{r}}\left[\frac{\dot{\phi}_{\boldsymbol{r}}^{2}(\tau)}{2 U}+\mathrm{i} \frac{\bar{\mu}}{U} \dot{\phi}_{\boldsymbol{r}}(\tau)\right],
$$

where $\dot{\phi}=\partial_{\tau} \phi$ and $\bar{\mu}=\mu+U / 2$, while

$$
\begin{aligned}
\mathcal{L}^{\prime} & =-t \sum_{\left\langle\boldsymbol{r}>\boldsymbol{r}^{\prime}\right\rangle}\left[\mathrm{e}^{-\mathrm{i} \phi_{\boldsymbol{r}}(\tau)+\mathrm{i} \phi_{\boldsymbol{r}^{\prime}}(\tau)} \bar{b}_{\boldsymbol{r}}(\tau) b_{\boldsymbol{r}^{\prime}}(\tau)+\text { H.c. }\right] \\
& +\sum_{\boldsymbol{r}}\left[\bar{b}_{\boldsymbol{r}}(\tau) \partial_{\tau} b_{\boldsymbol{r}}(\tau)+\left(U n_{\mathrm{B}}-\bar{\mu}\right) n_{\boldsymbol{r}}(\tau)\right] .
\end{aligned}
$$


Furthermore, we follow the method described in Ref. [27]. As a consequence of variable decomposition in Eq. (7), the order parameter factorizes

$$
\Psi \equiv\left\langle a_{\boldsymbol{r}}(\tau)\right\rangle=b_{0} \psi_{0},
$$

where $b_{0} \equiv\left\langle b_{\boldsymbol{r}}(\tau)\right\rangle$ is the Bogolyubov amplitude, which is non-vanishing in the strong-coupling limit at zero temperature and

$$
\psi_{0}=\left\langle\mathrm{e}^{\mathrm{i} \phi_{\boldsymbol{r}}(\tau)}\right\rangle,
$$

measures the phase coherence. Another consequence of Eq. (7) is factorisation of the $a$ - boson Green function of the system [28]:

$$
\text { with }
$$$$
G(\boldsymbol{r}, \tau) \equiv-\left\langle a_{\boldsymbol{r}}(\tau) \bar{a}(0)\right\rangle=-G_{\mathrm{B}}(\boldsymbol{r}, \tau) G_{\phi}(\boldsymbol{r}, \tau)
$$

$$
G_{\mathrm{B}}(\boldsymbol{r}, \tau)=-b_{0}^{2}-\left\langle b_{\boldsymbol{r}}^{\prime}(\tau) \bar{b}^{\prime}(0)\right\rangle
$$

and $b_{\boldsymbol{r}}^{\prime}(\tau)=b_{0}-b_{\boldsymbol{r}}(\tau)$. The Green function for the rotor field

$$
G_{\phi}(\boldsymbol{r}, \tau)=-\left\langle\mathrm{e}^{\mathrm{i}\left[\phi_{\boldsymbol{r}}(\tau)-\phi(0)\right]}\right\rangle,
$$

defines the value of the phase order parameter (11):

$$
1-\psi_{0}^{2}=\frac{1}{N \beta} \sum_{\boldsymbol{k}, m \neq 0} G_{\phi}\left(\boldsymbol{k}, \omega_{m}\right) .
$$

Performing analytic continuation of the Green functions one can obtain their spectral form

$$
A(\boldsymbol{k}, \omega)=\left.2 \operatorname{Im} G(\boldsymbol{k}, \omega)\right|_{\mathrm{i} \omega_{m} \rightarrow \omega+\mathrm{i} 0^{+}},
$$

which obeys the basic sum rule [29]:

$$
\frac{1}{N} \sum_{\boldsymbol{k}} \int_{-\infty}^{+\infty} \mathrm{d} \omega A(\boldsymbol{k}, \omega) n_{\mathrm{B}}(-\omega)=n_{a}
$$

with $n_{a}$ being the bosonic density. The spectral density of the Green function of the system in Eq. (12) is given by $[30,31]$ convolution of the amplitude and phase Green functions with additional factors $n_{\mathrm{B}}(\omega)=1 /\left(\mathrm{e}^{\beta \omega}-1\right)$ being the Bose-Einstein distribution

$$
\begin{aligned}
& A(\boldsymbol{k}, \omega)=-\frac{1}{N} \sum_{\boldsymbol{k}^{\prime}} \int_{-\infty}^{+\infty} \frac{\mathrm{d} \omega^{\prime}}{2 \pi}\left[n_{\mathrm{B}}\left(\omega^{\prime}\right)-n_{\mathrm{B}}\left(\omega^{\prime}-\omega\right)\right] \\
& \quad \times A_{\mathrm{B}}\left(\boldsymbol{k}^{\prime}, \omega^{\prime}\right) A_{\phi}\left(\boldsymbol{k}-\boldsymbol{k}^{\prime}, \omega-\omega^{\prime}\right)+\psi_{0}^{2} A_{\mathrm{B}}(\boldsymbol{k}, \omega) .(17)
\end{aligned}
$$

Integration over frequencies and momenta leads to a sum rule of the spectral function

$$
\frac{1}{N} \sum_{\boldsymbol{k}} \int_{-\infty}^{+\infty} \frac{\mathrm{d} \omega}{2 \pi} A(\boldsymbol{k}, \omega)=1 .
$$

This allows to calculate the spectral density $A_{S}(\boldsymbol{k}, \omega)$ of the structure factor, which can be written in a similar form using the single particle densities $A(\boldsymbol{k}, \omega)$ :

$$
\begin{aligned}
& A_{S}(\boldsymbol{k}, \omega)=\frac{1}{N} \sum_{\boldsymbol{k}^{\prime}} \int_{-\infty}^{+\infty} \frac{\mathrm{d} \omega^{\prime}}{2 \pi}\left[n_{\mathrm{B}}\left(\omega^{\prime}\right)-n_{\mathrm{B}}\left(\omega+\omega^{\prime}\right)\right] \\
& \quad \times A\left(\boldsymbol{k}^{\prime}, \omega^{\prime}\right) A\left(\boldsymbol{k}^{\prime}-\boldsymbol{k}, \omega^{\prime}+\omega\right) .
\end{aligned}
$$

As a result, the structure factor reads

$$
\begin{aligned}
& S(\boldsymbol{k}, \omega)=\frac{1}{N} \sum_{\boldsymbol{k}^{\prime}} \int_{-\infty}^{+\infty} \frac{\mathrm{d} \omega^{\prime}}{2 \pi} \frac{n_{\mathrm{B}}\left(\omega^{\prime}\right)-n_{\mathrm{B}}\left(\omega+\omega^{\prime}\right)}{1-\mathrm{e}^{-\beta \omega}} \\
& \quad \times A\left(\boldsymbol{k}^{\prime}, \omega^{\prime}\right) A\left(\boldsymbol{k}^{\prime}-\boldsymbol{k}, \omega^{\prime}+\omega\right) .
\end{aligned}
$$

Once again, a sum rule can be recovered

$$
\frac{1}{N} \sum_{\boldsymbol{k}} \int_{-\infty}^{+\infty} \frac{\mathrm{d} \omega}{2 \pi} S(\boldsymbol{k}, \omega)=n_{a}\left(1+n_{a}\right),
$$

which value is dependent only on the bosonic density.

\section{Numerical analysis and results}

Evaluation of the integrals in Eqs. (17) and (20) cannot be performed analytically and requires numerical calculations. The integration has to be performed over threedimensional space since the momentum $\boldsymbol{k}$ has two components and the frequency $\omega$ - one. Such operation performed directly would be extremely time consuming. To this end we lean on the convolution theorem, which states that convolution of two functions can be replaced by mixture of simple multiplication and Fourier transforms $\mathcal{F}$ :

$$
f * g=\mathcal{F}^{-1}[\mathcal{F}[f] \cdot \mathcal{F}[g]] .
$$

As computation of the Fourier transform is numerically relatively efficient, the convolutions can be calculated for reasonably high resolution in $k$ and $\omega$, with limits being imposed by the amount of available computer memory. For example, using $128 \mathrm{~GB}$ of RAM allows for about 5 billion division points. In the following we use a discretisation of the momentum-energy space into $1500 \times 1500 \times 1500$ divisions for $k_{x}, k_{y}$ and $\omega$, respectively. Our results are presented at $T=0$ limit, however the method used is not limited to this regime. Also, three-dimensional lattice could be used, however it would significantly reduce resolutions of the obtained spectral function and structure factor maps, since 5 billion division points would have to be distributed between three components.

Momentum and energy dependence of the singleparticle spectral function is presented in Figs. 1 and 2 . For bosons, the spectral function can be either positive or negative and, consequently, cannot be simply interpreted as probability density of exciting a particle with momentum $\boldsymbol{k}$ and energy $\boldsymbol{\omega}$, as in case of fermions. Sharp features in the inner edges of the spectra originate from the Bogolyubov quasiparticles, which are present for any values of $t / U$. In the superfluid state another sharp features appears signalling long range phase coherence of bosons. On the contrary, in the Mott insulating phase, a gap opens at zero momentum, leading to localisation of particles. The sharp features are accompanied with broad, smeared component of the spectrum, which results from presence of strong interactions between constituent incoherent particles. The spectral function cannot be directly observed in the Bragg spectroscopy, since it only registers two-particle excitations. It is however reminiscent of results of angle-resolved photoelectron spectroscopy (ARPES) experiments performed in solid state physics, which display similar behaviour of strongly correlated electronic systems: sharp features resulting from long-range ordering, broadened maxima following from the presence of strong interactions. 


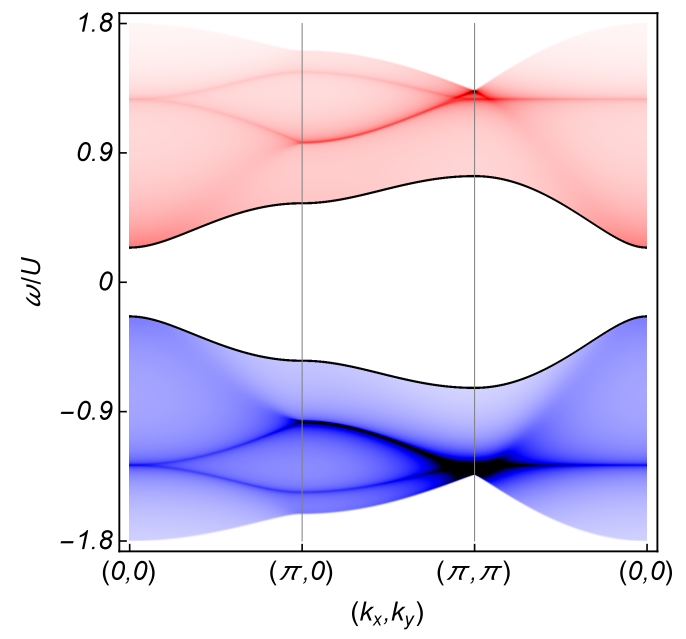

Fig. 1. Density plot of the one-particle spectral function $A(\boldsymbol{k}, \omega)$ in the Mott insulating phase presented in the energy-momentum space for $t / U=0.05, \mu / U=0.5$ along representative momentum cuts over the Brillouin zone: $\boldsymbol{k}=(0,0) \rightarrow(\pi, 0) \rightarrow(\pi, \pi) \rightarrow(0,0)$. Dark, sharp lines on the inner edge of the spectrum originate from the Bogolyubov quasiparticles. Higher intensity denotes larger absolute values of the spectral function, with $A(\boldsymbol{k}, \omega)$ being negative for $\omega>0$ (red) and positive for $\omega<0$ (blue). A gap opening around $\boldsymbol{k}=(0,0)$ point signals presence of the Mott insulating state. The broad incoherent background results from strong many-body effects.

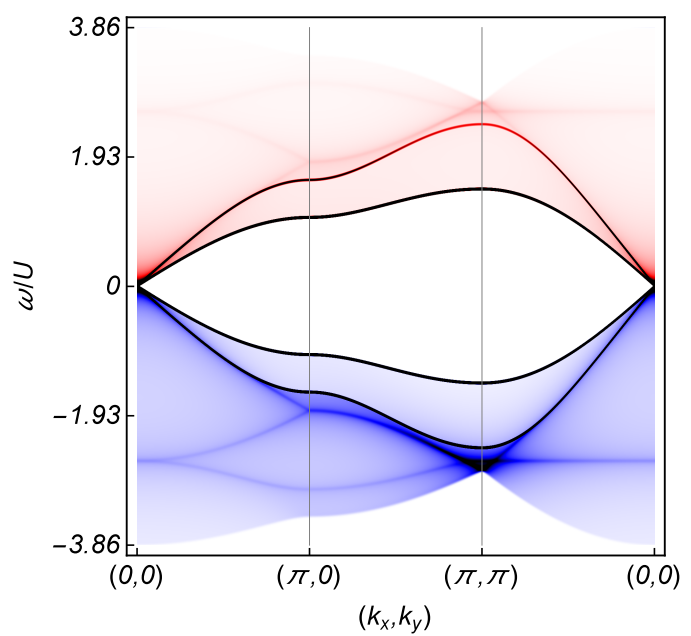

Fig. 2. Density plot of the one-particle spectral function $A(\boldsymbol{k}, \omega)$ in the superfluid phase presented in the energy-momentum space for $t / U=0.16, \mu / U=0.5$, along representative momentum cuts, as in Fig. 1. Dark, sharp lines on the inner edge of the spectrum originate from the Bogolyubov quasiparticles, while additional sharp features result from phase coherence of bosons. They both share linear excitation energy dependence on momentum around $\boldsymbol{k}=(0,0)$. Higher intensity denotes larger absolute values of the spectral function, with $A(\boldsymbol{k}, \omega)$ being negative for $\omega>0$ (red) and positive for $\omega<0$ (blue).

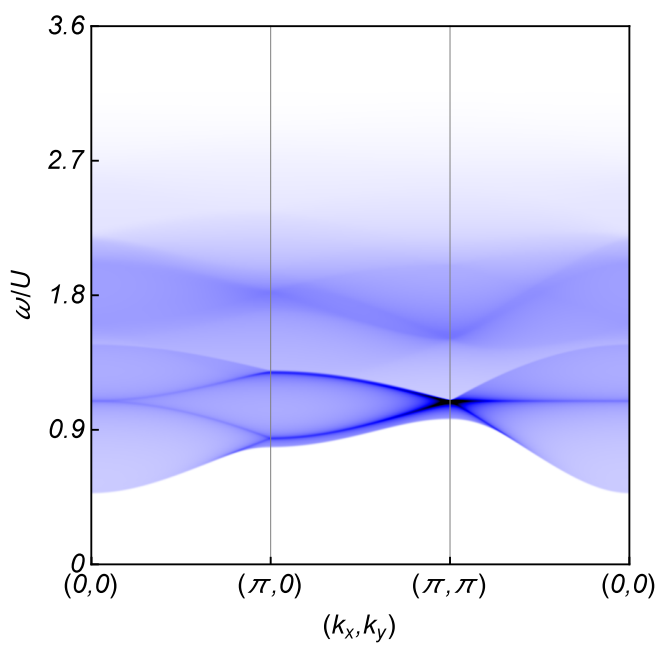

Fig. 3. Density plot of the momentum dependence of the dynamic structure factor $S(\boldsymbol{k}, \omega)$ in the Mott insulating state for $t / U=0.05, \mu / U=0.5$ along representative momentum cuts, as in previous figures. Higher intensity denotes larger values of the structure factor, which is always positive. A gap opening around $\boldsymbol{k}=(0,0)$ point signals presence of the Mott insulating state, while sharp features are missing.

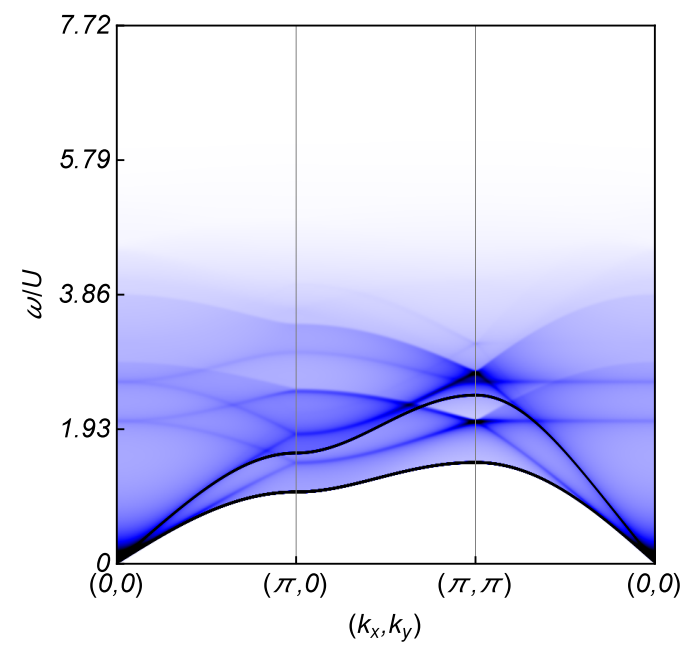

Fig. 4. Density plot of the momentum dependence of the dynamic structure factor $S(\boldsymbol{k}, \omega)$ in the superfluid state for $t / U=0.16, \mu / U=0.5$ along representative momentum cuts, as in previous figures. Higher intensity denotes larger values of the structure factor, which is always positive. Dark, sharp lines on the lower edge of the spectrum originate from the Bogolyubov quasiparticles, while additional sharp feature inside the smeared part of the spectrum is a condensate peak resulting from phase coherence of bosons. They both share linear excitation energy dependence on momentum around $\boldsymbol{k}=(0,0)$.

Knowledge of single-particle spectral function allows for calculation of dynamic structure factor using Eq. (20). Its momentum and energy dependence is depicted in Figs. 3 and 4 . In this case, sharp feature originating from the Bogolyubov particles is visible only for the superfluid state and in this case it occurs along the second 
one - coherence peak resulting from long-range phase coherence of bosons. However, it can be observed that transition from the MI to the SF state does not only close the zero-momentum gap, but also adds additional bands in the smeared component of the spectrum resulting from presence of strong interactions between particles. In both cases of Figs. 1 and 2 and Figs. 3 and 4, the sharp phase coherence peaks grow at the expense of the spectral weight of the incoherent part of the spectra. This is a necessary consequence of the sum rules which say that the total spectral weight of the dynamic structure factor $S(\boldsymbol{k}, \omega)$ and spectral function $A(\boldsymbol{k}, \omega)$ must be conserved and equal to $n_{a}\left(n_{a}+1\right)$ and 1 , respectively [see Eqs. (18) and (21)]. This might suggest a possible connection between the nature of the Mott state and the formation of sharply defined quasiparticle excitations in the superfluid. Also, it means that the superfluid state is built from states with wide range of energies.

\section{Conclusions}

In summary, we have presented a study of the dynamic structure factor for strongly interacting bosons, which is intrinsically connected with the one-particle spectral function. Using the fast Fourier transform we were able to compute multidimensional convolutions and obtain high-resolution spectra of these quantities. Both of them consist of sharp features, which originate from presence of the Bogolyubov quasiparticles or long-range phase coherence of the system and smeared components following from strong interactions between particles. The spectral weight transfer during the transition from the Mott insulator to the superfluid phase allows for observation on how the phase coherence is built in the system. At its current state of development the Bragg spectroscopy is not mature enough to present density maps of the momentum and energy-resolved dynamic structure factor in a manner similar to ARPES. However, we hope that our predictions will stimulate further development of experimental methods.

\section{References}

[1] I. Bloch, J. Dalibard, W. Zwerger, Rev. Mod. Phys. 80, 885 (2008).

[2] M. Greiner, O. Mandel, T. Esslinger, T.W. Hänsch, I. Bloch, Nature 415, 39 (2002).

[3] M. Aidelsburger, M. Atala, M. Lohse, J.T. Barreiro, B. Paredes, I. Bloch, Phys. Rev. Lett. 111, 185301 (2013).

[4] J. Struck, M. Weinberg, C. Ölschläger, P. Windpassinger, J. Simonet, K. Sengstock, R. Höppner, P. Hauke, A. Eckardt, M. Lewenstein, L. Mathey, Nat. Phys. 9, 738 (2013).

[5] S. Krinner, D. Stadler, D. Husmann, J.-P. Brantut, T. Esslinger, Nature 517, 64 (2015).
[6] W.S. Bakr, J.I. Gillen, A. Peng, S. Fölling, M. Greiner, Nature 462, 77 (2009).

[7] J.F. Sherson, C. Weitenberg, M. Endres, M. Cheneau, I. Bloch, S. Kuhr, Nature 467, 68 (2010).

[8] G.K. Campbell, J. Mun, M. Boyd, P. Medley, A.E. Leanhardt, L.G. Marcassa, D.E. Pritchard, W. Ketterle, Science 313, 649 (2006).

[9] J.T. Stewart, J.P. Gaebler, D.S. Jin, Nature 454, 744 (2008).

[10] T.-L. Dao, A. Georges, J. Dalibard, C. Salomon, I. Carusotto, Phys. Rev. Lett. 98, 240402 (2007).

[11] J. Stenger, S. Inouye, A.P. Chikkatur, D.M. StamperKurn, D.E. Pritchard, W. Ketterle, Phys. Rev. Lett. 82, 4569 (1999).

[12] A.M. Ray, P.B. Blakie, G. Pupillo, C.J. Williams, C.W. Clark, Phys. Rev. A 72, 023407 (2005).

[13] D. Clément, N. Fabbri, L. Fallani, C. Fort, M. Inguscio, J. Low. Temp. Phys. 158, 5 (2010).

[14] P.T. Ernst, S. Götze, J.S. Krauser, K. Pyka, D.-S. Lühmann, D. Pfannkuche, K. Sengstock, Nat. Phys. 6, 56 (2009).

[15] P. Pippan, H.G. Evertz, M. Hohenadler, Phys. Rev. A 80, 033612 (2009).

[16] V.N. Golovach, A. Minguzzi, L.I. Glazman, Phys. Rev. A 80, 043611 (2009).

[17] G. Pupillo, A.M. Rey, G.G. Batrouni, Phys. Rev. A 74, 013601 (2006)

[18] S. Ejima, H. Fehske, F. Gebhard, K. zu Münster, M. Knap, E. Arrigoni, W. von der Linden, Phys. Rev. A 85, 053644 (2012).

[19] G. Roux, A. Minguzzi, T. Roscilde, New J. Phys. 15, 055003 (2013).

[20] T.P. Polak, T.K. Kopeć, Phys. Rev. B 76, 094503 (2007).

[21] A. Brynello, D. Dalfovo, L. Pitaevskii, S. Stringari, F. Zambelli, Phys. Rev. A 64, 063614 (2001).

[22] D. Pines, P. Nozieres, The Theory of Quantum Liquids, Vol. I, Benjamin, New York 1966.

[23] A.L. Fetter, J.D. Walecka, Quantum Theory of ManyParticle Systems, McGraw-Hill, San Francisco 1971.

[24] M.P.A. Fisher, P.B. Weichman, G. Grinstein, D.S. Fisher, Phys. Rev. B 40, 546 (1989).

[25] D. Jaksch, C. Bruder, J.I. Cirac, C.W. Gardiner, P. Zoller, Phys. Rev. Lett. 81, 3108 (1998).

[26] S. Florens, A. Georges, Phys. Rev. B 66, 165111 (2002).

[27] T.A. Zaleski, T.K. Kopeć, Phys. Rev. A 84, 053613 (2011).

[28] C. Lannert, M.P.A. Fisher, T. Senthil, Phys. Rev. B 64, 014518 (2001).

[29] C. Menotti, N. Trivedi, Phys. Rev. B 77, 235120 (2008).

[30] T.A. Zaleski, Phys. Rev. A 85, 043611 (2012).

[31] T.A. Zaleski, T.K. Kopeć, Physica B 433, 37 (2014). 